\title{
ATRIBUTOS ECOLÓGICOS Y DE HÁBITAT DE LAS ESPECIES SUCULENTAS DEL ÁREA NATURAL PROTEGIDA MONUMENTO NATURAL YAGUL, OAXACA, MÉXICO
}

\section{ECOLOGICAL AND HABITAT ATTRIBUTES OF THE SUCCULENT SPECIES OF THE NATURAL PROTECTED AREA, NATURAL MONUMENT YAGUL, OAXACA, MEXICO}

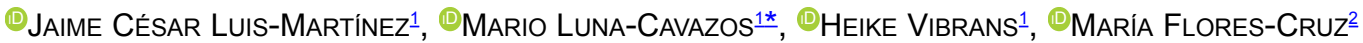

1Posgrado en Botánica, Colegio de Postgraduados, Campus Montecillo, Montecillo, Estado de México, México.

2División de Ciencias Biológicas y de la Salud, Universidad Autónoma Metropolitana-Unidad Xochimilco, Ciudad de México, México.

*Autor para correspondencia: mluna@colpos.mx

\section{Resumen}

Antecedentes: Las suculentas son especies características de ecosistemas secos. De los caracteres ambientales de sitio, las laderas, por ser áreas de escurrimiento tienden a estar más secas; allí se establecen suculentas de importancia ecológica.

Hipótesis: No existen diferencias en la composición florística y atributos ecológicos de suculentas, en laderas de distinta orientación del Monumento Natural Yagul.

Descripción de datos: densidad, cobertura y frecuencia; variables edáficas.

Sitio de estudio: Yagul, Tlacolula, Oaxaca a 1,600 m snm. Las coordenadas geográficas $16^{\circ} 58^{\prime} 26^{\prime \prime}$ y $16^{\circ} 55^{\prime} 56^{\prime \prime}$ Norte, $96^{\circ} 28^{\prime} 17^{\prime \prime}$ y $96^{\circ}$ 25' 51" Oeste.

Métodos: Cálculo de valores de importancia. Mediante métodos multivariables, se comparó la semejanza florística entre laderas, y se evaluaron variables topográficas y edáficas relacionadas con la distribución de las especies.

Resultados: La riqueza florística de suculentas en Yagul, fue de 21 especies; las de mayor IVI fueron Opuntia pubescens presente en todas las orientaciones de ladera, Hechtia pringlei en la ladera norte, O. pilifera en la sur y Myrtillocactus schenckii en las laderas este y oeste. Las laderas del norte presentaron la mayor diferencia florística respecto al resto. Las variables edáficas más importantes, para la distribución de poblaciones, fueron el contenido de materia orgánica y el fósforo.

Conclusiones: Existe diferencia en la composición florística entre laderas; los sitios de la ladera norte, tiene la menor riqueza y se diferencian del resto. Los sitios de la ladera sur tienen mayor contenido de materia orgánica y fósforo, que favorece especies como Stenocereus treleasei y S. stellatus.

Palabras clave: Análisis multivariado, laderas, riqueza, semejanza florística, variables edáficas.

\begin{abstract}
Background: Succulents are characteristic species of dry ecosystems. Of the environmental characters of the site, the slopes are runoff areas and they tend to be even drier. On the slopes succulent species of economic and ecological importance are established.

Hypothesis: There are no differences in the floristic composition and ecological attributes of succulents on the slopes of different orientation at the Yagul Natural Monument.

Data description: density, coverage and frequency; edaphic variables.

Study site: Yagul, Tlacolula, Oaxaca a 1,600 m asl. Geographical coordinates $16^{\circ} 58^{\prime} 26^{\prime \prime}$ y $16^{\circ} 55^{\prime} 56^{\prime \prime}$ N, $96^{\circ} 28^{\prime} 17^{\prime \prime}$ y $96^{\circ} 25^{\prime} 51^{\prime \prime}$ W.

Methods: Calculation of importance values. Through multivariate methods the floristic similarity among slopes was compared, and topographic and edaphic variables related to the species distribution were analyzed.

Results: The richness of succulents in Yagul was 21 species; those with the highest IVI were Opuntia pubescens present in all slope orientations, Hechtia pringlei on the north slope, O. pilifera in the south and Myrtillocactus schenckii on the east and west slopes. The northfacing slopes showed the greatest floristic difference from the rest. The most important edaphic variables in the distribution of populations were the content of organic matter and phosphorus.

Conclusions: There is a difference in the floristic composition between slopes; the sites on the northern slope have the lowest richness and differ from the rest. The sites of the southern slope have a higher content of organic matter and phosphorus, which favors species such as Stenocereus treleasei and S. stellatus.
\end{abstract}

Keywords: Edaphic variables, floristic similarity, multivariate analysis, richness, slopes. 
Las zonas secas en México presentan ecosistemas únicos por las condiciones extremas que prevalecen. Estas características han propiciado la persistencia de muchas especies endémicas, por lo cual, se consideran de alta importancia para su conservación (Rzedowski 1978). Las condiciones ambientales de baja humedad y sequía en los Valles Centrales del estado de Oaxaca, han determinado en gran parte que la vegetación contenga caracteres xerofíticos. Las familias suculentas, mejor representadas son, Cactaceae, Asparagaceae y Bromeliaceae.

El Monumento Natural Yagul, es un área arqueológica, situada dentro del Área Natural Protegida (ANP), actualmente Patrimonio Cultural de la Humanidad, por parte de la Organización de las Naciones Unidas para la Educación, la Ciencia y la Cultura (UNESCO). La zona alberga especies útiles asociadas a las costumbres alimentarias de los antiguos pobladores de la región de los Valles Centrales de Oaxaca. De los magueyes (Agave spp.) se aprovechaba el tallo por la producción de aguamiel; del mezquite (Prosopis juliflora (Sw.) DC.) la miel de las flores y del nopal (Opuntia spp.), los cladodios y frutos. En el Valle de Oaxaca, en la zona de Yagul y Mitla (zona cercana al Monumento Natural) se encuentran las evidencias del uso y aprovechamiento desde tiempos prehispánicos (CONABIO 2008).

El sitio, fisonómicamente destaca por laderas rocosas con pendientes suaves o abruptas y ecosistemas secos. En la actualidad, Yagul y los territorios aledaños evidencian deterioro, y aun así, esta ANP funciona como un refugio de la flora y fauna local, así como fuente y reserva de germoplasma nativo de la región (SEMARNAT 2013). Yagul está situado en los límites de la Provincia Florística de las Serranías Meridionales, Valle de Tehuacán y Costa Pacífica, representa características peculiares que lo sitúan como zona importante para la conservación, de acuerdo con la Comisión Nacional para el Conocimiento y Uso de la Biodiversidad (CONABIO 2008).

Se han realizado diversos estudios de flora y fauna en Oaxaca, pero, actualmente no se conoce la vegetación del Monumento Natural de Yagul. Hecho que motiva a generar bases de conocimiento sobre los recursos presentes y el estado ecológico, con el fin de que puedan generarse esquemas de conservación a futuro.

La orientación de la ladera modifica las condiciones microclimáticas de los sitios. A pesar de este conocido patrón son pocas las evidencias de su efecto sobre las poblaciones de plantas en regiones tropicales y son aún menos conocidas para las suculentas (López-Gómez et al. 2012). Reyes-Olivas et al. (2002) identificaron un gradiente ecológico asociado con la composición vegetal, el factor de sequía determinado por la exposición de ladera, la profundidad del suelo y la cobertura de rocas. Mazzola et al. (2008) mencionan que las características ambientales vinculadas a la posición en el relieve y los cambios topográficos leves o moderados afectan de manera significativa la distribución espacial y abundancia de las plantas vasculares. En el Valle de Zapotitlán, Puebla, los arbustos dominan las laderas norte (Valverde-Padilla 2002); en las laderas sur abundan las plantas rosetófilas y hay mayor riqueza de formas de vida. En nopaleras de diferentes exposiciones en Zacatecas, Opuntia domina en todos los sitios, pero los orientados al norte carecen de especies existentes en el lado sur (Del Castillo 2000). Otro caso es el de la especie Ferocactus histrix (DC.) G.E. Linds., que presentó acentuadas diferencias en su estructura poblacional en sitios cercanos con condiciones contrastantes debido a la orientación de laderas (Del Castillo 1987). La evaluación de la vegetación de una montaña desértica del sureste de los Estados Unidos y norte de México por efecto de orientación y elevación de los sitios, demostró que la densidad de plantas suculentas fue mayor en la parte baja en exposiciones sur y oeste (Mata-González et al. 2002). En otros entornos como en las Sierras de Lihue Calel, Argentina, también hay efectos de orientación de las laderas, se ve mayor número de comunidades vegetales hacia las laderas norte en comparación con las del sur (Mazzola et al. 2008).

El presente trabajo tiene como propósito analizar los factores topográficos y edáficos que influyen en la composición y atributos ecológicos de la flora de suculentas de ladera, en la región de los Valles Centrales de Oaxaca, México. La hipótesis que se planteó en esta investigación fue, que no existen diferencias en la composición florística y atributos ecológicos de suculentas en las laderas de distinta orientación del Monumento Natural Yagul, Oaxaca.

La información obtenida, permitirá conocer las condiciones físicas, requeridas para la sobrevivencia y persistencia de las especies de suculentas. Desde el punto de vista de acciones, se podrá contribuir a fijar metas que coadyuven al cuidado y conservación de las especies suculentas, especialmente de aquéllas con limitados atributos ecológicos.

\section{Materiales y métodos}

Área de estudio. Yagul se ubica en el municipio de Tlacolula de Matamoros, a 1,600 m snm, en la parte central del estado de Oaxaca, México. Las coordenadas geográficas de la zona de estudio son $16^{\circ} 58^{\prime} 26^{\prime \prime}$ a $16^{\circ} 55^{\prime} 56^{\prime \prime} \mathrm{N}, 96^{\circ}$ $28^{\prime} 17^{\prime \prime}$ a $96^{\circ} 25^{\prime} 51^{\prime \prime}$ O (Figuras 1 y 2 ). El clima es semiseco-semicálido como lo describe el Instituto Nacional de Estadística, Geografía e Informática (INEGI 2000) o semiárido templado cálido (BS1kw) de acuerdo con García (2004). La precipitación y temperatura promedio anual es de $700 \mathrm{~mm}$ y $18{ }^{\circ} \mathrm{C}$, respectivamente (INEGI 2005). Los tipos de vegetación existentes son Selva Baja Caducifolia y 
Selva Baja Caducifolia Espinosa (Miranda \& Hernández X. 2013).

Muestreo de vegetación. El trabajo de campo se llevó a cabo en el período de agosto de 2017 a mayo de 2018. Se trabajó en cuatro áreas montañosas (Figura 2), sobre las laderas con orientaciones al norte, sur, este y oeste. Con base en un reconocimiento exploratorio del área de estudio, en cada ladera se siguió un muestreo sistemático; se establecieron seis unidades de muestreo (UM) las cuales se ubicaron donde se permitió el acceso al ANP. Este procedimiento se basó en las dimensiones del área de estudio y en la fisonomía de las asociaciones vegetales de cada ladera. Cada UM estuvo constituida por tres transectos de $30 \times 2 \mathrm{~m}$

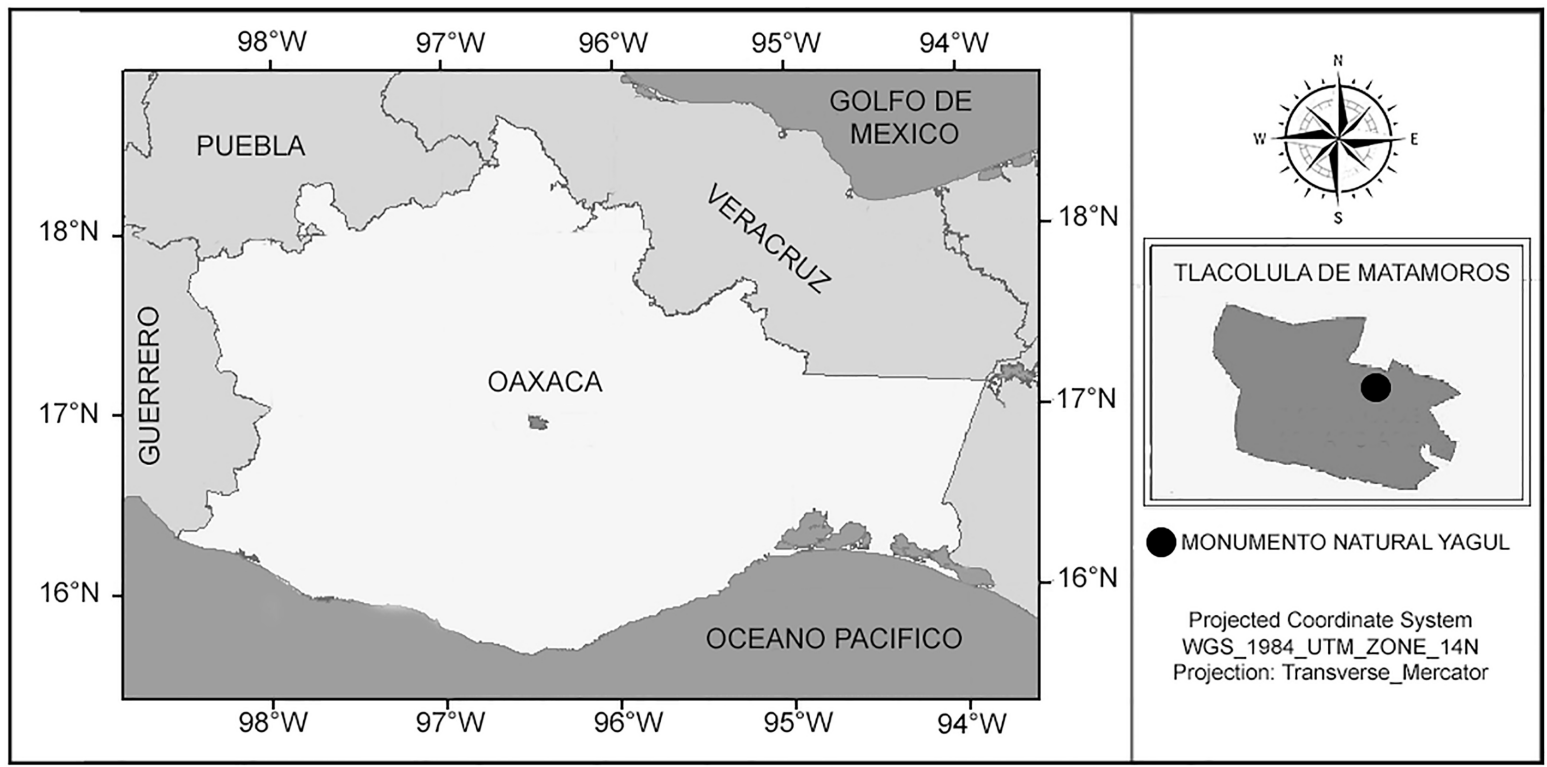

Figura 1. Zona de estudio, Monumento Natural Yagul, Tlacolula, Oaxaca.

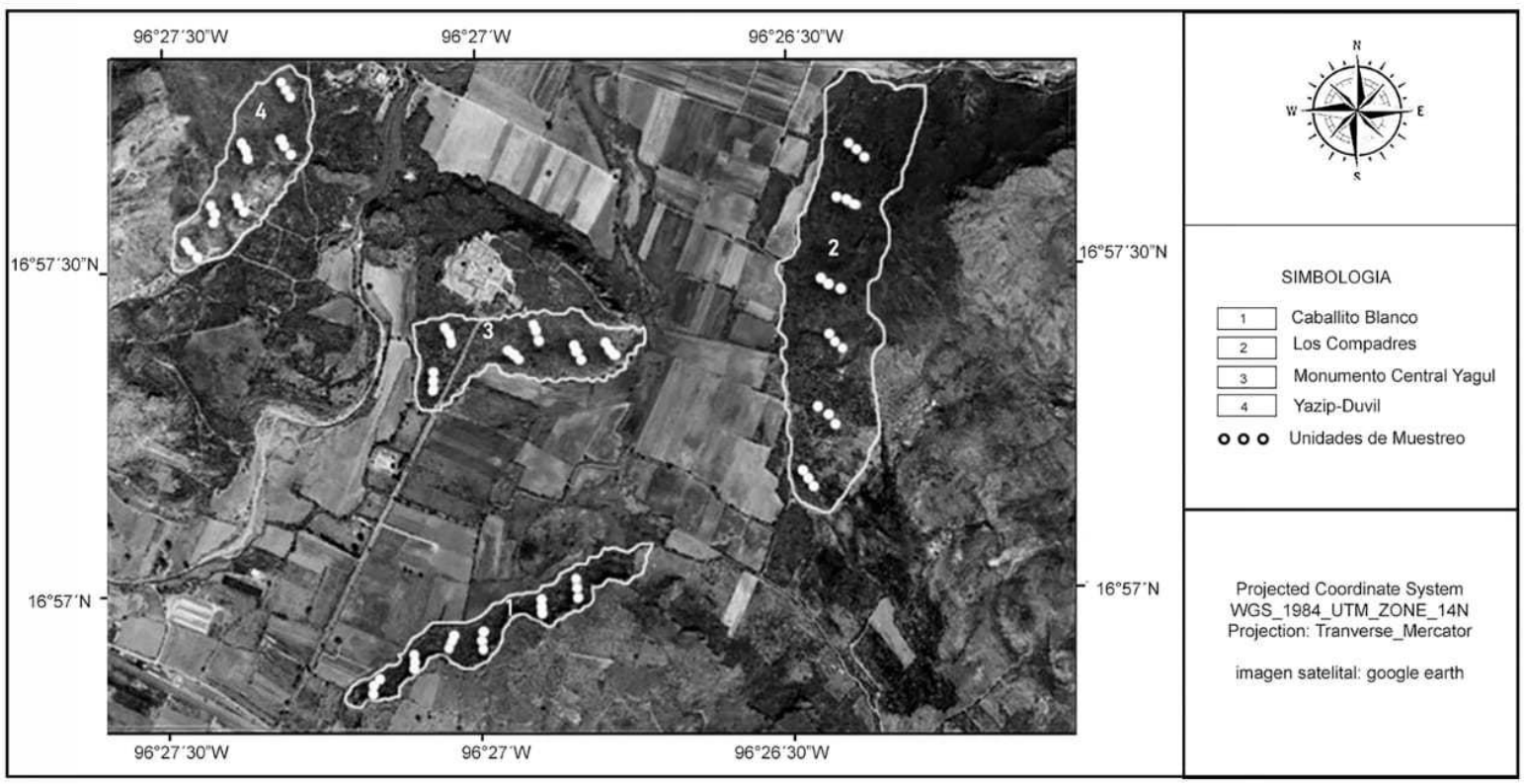

Figura 2. Mapa de las Unidades de Muestreo 1) Caballito Blanco 2) Los Compadres 3) Monumento Central Yagul y 4) Yazip-Duvil. Google maps. Recuperado el 3 de noviembre, 2018. 
(Figura 3), distribuidos al azar en la parte baja, media y alta de la ladera, para hacer un total de 18 transectos por ladera y 72 para toda el área de estudio; el propósito fue abarcar la mayor superficie posible del área. En las UM se preidentificaron las especies de suculentas, se contaron los individuos por superficie para calcular la densidad, se midió la amplitud de copa de cada individuo para estimar la cobertura y se contó el número de sitios donde se registró cada especie con respecto al total, para definir la frecuencia de aparición. Se recolectaron muestras de material botánico, el cual fue procesado mediante las técnicas convencionales (Lot \& Chiang 1986). Las especies se identificaron con el apoyo de bibliografía especializada tales como claves y descripciones de floras regionales, así como, de revisiones y monografías de grupos taxonómicos. El material biológico colectado fue depositado en el Herbario-Hortorio (CHAPA) del Colegio de Postgraduados. La nomenclatura taxonómica se cotejó en el sitio Tropicos del Missouri Botanical Garden (http://www.tropicos.org) y la asignación de las familias, se determinó con base en la clasificación APG, Grupo IV para la Filogenia de las Angiospermas - Angiosperm Phylogeny Group IV (APG 2016).

Muestras de suelo. Se recolectaron muestras de suelo, para analizar la relación de algunas variables físico-químicas edáficas con los patrones de distribución de las suculentas del área de estudio. Se extrajeron 24 muestras, cada una conformada por tres submuestras compuestas, obtenidas de los tres transectos que constituyeron la UM, distribuidos en la parte baja, media y alta de las laderas. Las variables edáficas consideradas y el método para su medición fueron: la textura mediante hidrómetro de Bouyoucos; el pH con un potenciómetro en relación suelo-agua; la materia orgánica (Walkley \& Black 1934); el fósforo se cuantificó con el método Olsen; la conductividad eléctrica (CE) se midió en una suspensión de agua del suelo con base en la Norma Oficial Mexicana (SEMARNAT 2002); el nitrógeno total se usó el método Kjeldahl, para ello, una muestra de suelo se digirió con ácido sulfúrico-salicílico, seguido de un análisis colorimétrico (Bremmer \& Mulvaney 1982); el potasio (K) y el carbonato de calcio $\left(\mathrm{CaCO}_{3}\right)$ se extrajeron con agua destilada y su concentración se determinó por titulación ácida.

Análisis de datos. Los datos de los atributos ecológicos estimados por cada especie (densidad, frecuencia y dominancia) se usaron para calcular el Índice de Valor de Importancia (IVI) de las especies (Mostacedo \& Fredericksen 2000).

La semejanza florística entre las distintas laderas, se evaluó mediante un análisis de agrupamiento de dos vías (Peck 2010), el cual se basó en el registro de 21 especies de suculentas en laderas orientadas en cuatro diferentes puntos cardinales. La base de datos se elaboró con el registro de presencia (1) o ausencia (0) de las especies en las diferentes UM de cada ladera. La matriz de datos se formó de 24 hileras (UM) y 21 columnas (especies). El análisis de las afinidades florísticas entre laderas inició con la estimación del índice de semejanza mediante el coeficiente de Jaccard. Con base en los valores de semejanza entre UM, se formaron los grupos con el método Flexible Beta, los cuales posteriormente se representaron en un dendrograma. Para

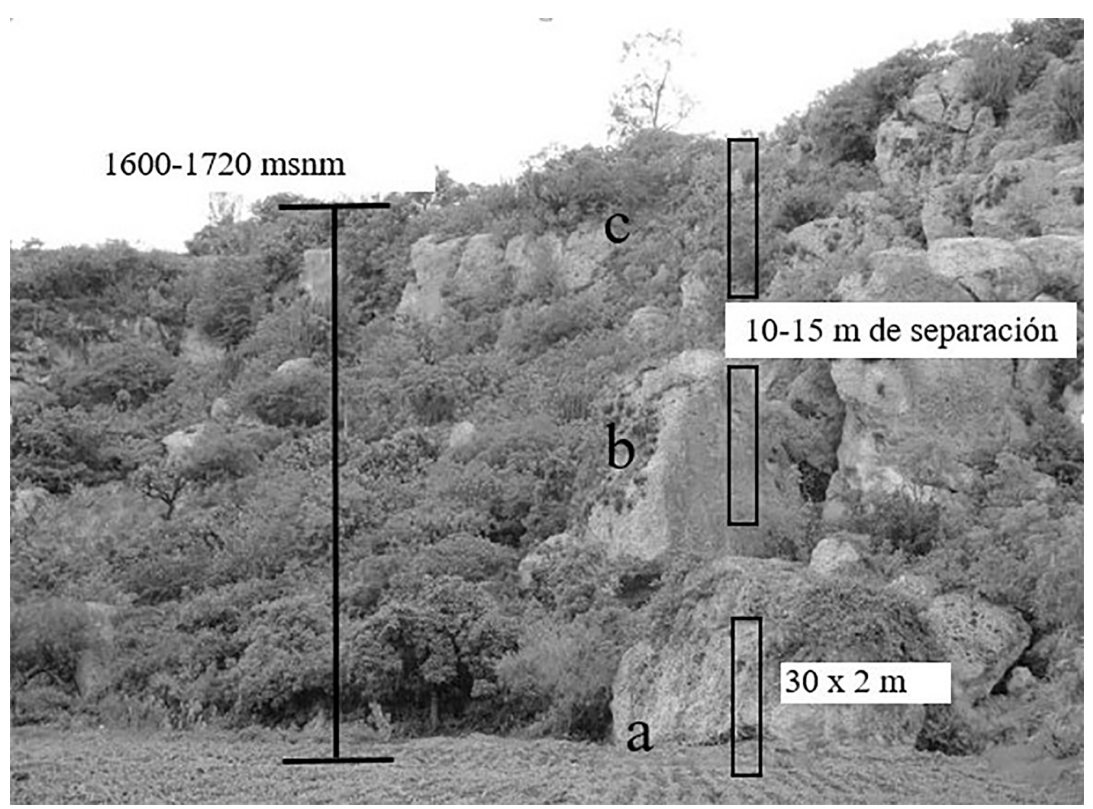

Figura 3. Esquema representativo de una Unidad de Muestreo (UM), constituida por tres transectos de $30 \times 2 \mathrm{~m}$; a) Parte baja de ladera; b) Parte media de ladera y c) Parte alta de ladera. Sitio "Caballito Blanco". 
determinar diferencias estadísticas en la composición florística entre laderas, se aplicó el procedimiento de permutación de respuesta múltiple (PPRM), de acuerdo con Mielke (1991) y Mielke \& Berry (1976).

La evaluación de la importancia de los factores edáficos, en la composición florística y distribución de las especies entre laderas, se llevó a cabo mediante análisis de ordenación. En el procesamiento de los datos, se excluyó del análisis a la textura del suelo por presentar poca variación. El análisis se basó en siete variables edáficas $(\mathrm{pH}$, conductividad eléctrica $\mathrm{CE}$, materia orgánica $\mathrm{MO}$, Nitrógeno total Nt, Fósforo P, Potasio K y Carbonato de Calcio $\mathrm{CaCO}_{3}$ ); las cuales fueron transformadas a logaritmo de acuerdo con las recomendaciones de McCune \& Grace (2002). Para el análisis se elaboraron dos matrices de datos. La matriz sitios-especies consistió de las $24 \mathrm{UM}$ y los valores de densidad de las 21 especies registradas; la segunda matriz, sitios-suelos, incluyó a las mismas unidades de muestreo y las variables edáficas analizadas. Con la primera matriz se llevó a cabo un análisis de correspondencia rectificado (AC) con el propósito de reconocer los patrones de asociación entre las UM y especies. Después, ambas matrices fueron relacionadas mediante un Análisis Canónico de Correspondencia (ACC), de acuerdo con el procedimiento de Ter Braak (1986). Los análisis de agrupamiento, PPRM y ordenación se efectuaron con el programa PC-ORD versión 6 (McCune \& Mefford 2011).

\section{Resultados}

Descripción de la riqueza y diversidad de especies en las diferentes laderas. Se registraron tres familias de suculentas en el área de estudio, Cactaceae, Asparagaceae y Bromeliaceae. La familia con mayor número de especies fue Cactaceae (11), de la que se registraron 1,033 individuos.

Laderas norte.- En ellas se registraron 13 especies de suculentas. Las que presentaron los mayores valores de IVI fueron Opuntia pubescens H.L. Wendl. ex Pfeiff. y Hechtia pringlei B.L. Rob. \& Greenm y Myrtillocactus schenckii (J.A. Purpus) Britton \& Rose. (Tabla 1). Se registró la presencia de dos epífitas: Tillandsia fasciculata Sw. y Tillandsia dasyliriifolia Baker que tienen la preferencia de crecer sobre los garambullos (M. schenckii y en formaciones rocosas. Agave convallis Trel. tuvo un IVI de 37.66, mucho más alto que en las demás laderas, donde los valores fueron menos de 4 . Hubo puntos de muestreo donde se llegaron a ver grandes poblaciones en partes medias de la ladera (Figura 3). La única suculenta globosa registrada fue Mammillaria karwinskiana Mart. con un valor de importancia de 7.06.
Laderas sur.- Se documentaron 15 especies. Los valores de importancia más altos pertenecieron a las especies $O$. pubescens seguida de Opuntia pilifera F.A.C. Weber (Tabla 1). Agave karwinskii Zucc. tuvo un IVI de 34.50 en la parte baja, se localizó, en los límites de la selva baja con los terrenos de sembradíos, donde forma parte de cercos vivos. Formas biológicas frecuentes en estas laderas, fueron las cactáceas columnares y candelabriformes. En la investigación, se registró un ejemplar joven de Ferocactus latispinus (Haw.) Britton \& Rose. También había algunos espacios dominados por los taxa Opuntia velutina F.A.C. Weber y M. schenckii.

Laderas este.- Se encontraron 18 especies de suculentas y las dominantes por su valor de importancia fueron M. schenckii y O. pubescens (Tabla 1). En estas laderas fue donde se registraron más especies. Del grupo de las biznagas, F. latispinus y Coryphantha retusa (Pfeiff.) Britton \& Rose presentaron los IVI más elevados (Tabla 1). Otra especie que se registró localmente fue Yucca periculosa Baker, no fue una especie abundante, se distribuía en lugares perturbados de actividades humanas. Se encontraron poblaciones densas de $C$. retusa en esta ladera en áreas muy específicas, intercaladas en la selva baja espinosa de Prosopis, colindantes con terrenos de sembradío.

Laderas oeste.- En estos sitios la riqueza es de 14 especies, de las cuales $O$. pubescens, $M$. schenckii y A. karwinskii fueron las de mayor IVI (Tabla 1). Opuntia pubescens y $M$. schenckii son dos de las especies con mayor dominancia en esta y las diferentes laderas. Es importante mencionar que el género Opuntia estuvo presente en todas las unidades de muestreo. Una especie que resaltó por su densidad fue Agave potatorum Zucc., del que encontramos una preferencia por ambientes muy secos, suelos someros y pedregosos, y presencia en diferentes altitudes, lo que hace pensar que es una de las especies mejor adaptadas a la zona seca de Yagul.

Semejanza florística. El análisis de semejanza florística entre laderas, diferenció dos grupos (G) principales (Figura 4). El primer grupo (G1) incluyó las laderas norte y la mayor parte de las orientadas al sur. Dentro de este grupo, se nota, la estrecha asociación de todas las UM orientadas al norte (SG1), en el cual las especies que lo caracterizan fueron: $O$. pubescens, $O$. velutina, O. pilifera, $M$. schenckii, H. pringlei, A. convallis y dos epífitas $T$. fasciculata y T. dasyliriifolia. Es importante aludir la separación de las UM de la ladera norte, con respecto al resto de las laderas, y aun cuando las UM del norte se incluyen en un grupo mayor con UM de otras laderas, la semejanza florística con estas es baja a juzgar por la distancia entre ellas. El segundo subgrupo (SG2) estuvo formado por cinco de seis 
Tabla 1. Índice de valor de importancia de las especies suculentas en las diferentes laderas de Yagul, Oaxaca. LN, ladera norte; LS, ladera sur; LE, ladera este; LO, ladera oeste

\begin{tabular}{|c|c|c|c|c|}
\hline Especie & $\mathbf{L N}$ & $\mathbf{L S}$ & $\mathbf{L E}$ & LO \\
\hline Agave angustifolia Haw. & 1.42 & & & \\
\hline Agave convallis Trel. & 37.66 & 4.41 & 1.05 & 2.77 \\
\hline Agave karwinskii Zucc. & 1.54 & 34.50 & 1.05 & 42.94 \\
\hline Agave marmorata Roezl & & & 5.77 & 21.78 \\
\hline Agave potatorum Zucc. & & 1.49 & 21.83 & 14.79 \\
\hline Coryphanta retusa (Pfeiff.) Britton \& Rose & & 4.71 & 9.46 & 2.98 \\
\hline Ferocactus latispinus (Haw.) Britton \& Rose & & 1.46 & 10.57 & 7.66 \\
\hline Hechtia pringlei B.L. Rob. \& Greenm. & 53.60 & 1.48 & 8.44 & 32.20 \\
\hline Hechtia sp. & 7.37 & & 2.77 & \\
\hline Myrtillocactus schenckii (J.A. Purpus) Britton \& Rose & 40.53 & 23.16 & 89.41 & 49.82 \\
\hline Mammillaria karwinskiana Mart. & 7.06 & 21.61 & 30.56 & 11.39 \\
\hline Neobuxbaumia sp. & & & 1.14 & \\
\hline Opuntia pilifera F.A.C. Weber & 12.46 & 62.06 & 9.41 & 20.76 \\
\hline Opuntia pubescens H.L.Wendl. ex Pfeiff. & 86.79 & 94.56 & 85.20 & 65.01 \\
\hline Opuntia velutina F.A.C. Weber & 13.10 & 29.62 & 12.55 & 15.62 \\
\hline Stenocereus pruinosus (Otto ex Pfeiff.) Buxb. & 3.96 & & 1.05 & 4.09 \\
\hline Stenocereus stellatus (Pfeiff.) Riccob. & & 5.12 & & \\
\hline Stenocereus treleasei (Vaupel) Backeb. & & 1.63 & & \\
\hline Tillandsia dasyliriifolia Baker & 19.28 & 9.38 & 2.51 & \\
\hline Tillandsia fasciculata Sw. & 15.17 & 4.72 & 2.53 & 8.12 \\
\hline Yucca periculosa Baker & & & 4.59 & \\
\hline
\end{tabular}

UM ubicadas en la ladera sur, contiguas tanto del este como del oeste, donde algunas de las especies más comunes fueron: $O$. pubescens, $M$. schenckii, $O$. velutina, $O$. pilifera y $H$. pringlei. Este subgrupo comparte con el anterior, diversas especies de las ya mencionadas, sin embargo, una diferencia está dada por la presencia de Stenocereus stellatus (Pfeiff.) Riccob. y la ausencia de las especies $S$. pruinosus, Hechtia sp. y A. angustifolia en SG2.

El G2 integra a las UM de laderas orientadas al este y oeste, éstas comparten muchas de las especies encontradas en otras laderas como: $O$. pubescens, M. schenckii, $O$. velutina, $O$. pilifera, $H$. pringlei, A. karwinskii, A. potatorum. Diferencias notables respecto a otras laderas principalmente del norte, fue la presencia de $A$. karwinskii, A. potatorum, Agave marmorata Roezl y poblaciones importantes de F. latispinus.

La prueba de permutación de respuesta múltiple indicó diferencias significativas $(p<0.05)$ en la composición de especies entre laderas (Tabla 2) este-oeste, norte-este y norte-sur. Con base en lo anterior, puede decirse que es diferente la composición de especies entre ellas. El estadístico $\mathrm{T}$ mostró la separación entre los grupos; los valores menores de $\mathrm{T}$ con signo negativo indican mayores diferencias entre ellos.

Tabla 2. Diferencias en la composición florística entre las diferentes laderas de Yagul, Oaxaca. LN: ladera norte, LE: ladera este, LO: ladera oeste, S: ladera sur

\begin{tabular}{lcc}
\hline Comparación & $\mathbf{T}$ & $\mathbf{P}$ \\
\hline LN vs LS & -3.6317 & 0.0088 \\
LN vs LE & -4.2832 & 0.0046 \\
LN vs LO & -3.3455 & 0.0079 \\
LS vs LE & -2.5191 & 0.0294 \\
LS vs LO & -3.3940 & 0.0081 \\
LE vs LO & -6.3871 & 0.0002 \\
\hline
\end{tabular}

Análisis de correspondencia. La asociación de los sitios y especies se presenta en la Figura 5. Los tres primeros ejes de la ordenación explican el $43 \%$ de la varianza total. La 
longitud del gradiente (LG) del eje uno fue de 3.82 lo que indica diferencias en la composición de especies entre laderas (Hill \& Gauch 1980), caso semejante para el eje dos con una LG de 3.15. La gráfica derivada de este análisis representa en cierta medida la asociación obtenida con la clasificación; principalmente las correspondientes a las laderas con orientación norte, las cuales muestran una diferenciación en el eje uno, asociadas fundamentalmente a especies de Agave, Hechtia y Tillandsia.
Análisis canónico de correspondencias (ACC). El ACC que relacionó las poblaciones de suculentas con las variables edáficas, indica que los 3 primeros ejes de ordenación explican $30.3 \%$ de la variación total. El primer eje es un gradiente definido por contenido de fósforo (0.745), materia orgánica (0.655) y conductividad eléctrica (0.591); en tanto que el eje dos estuvo caracterizado básicamente por el $\mathrm{pH}$ (0.546) (Tabla 3).
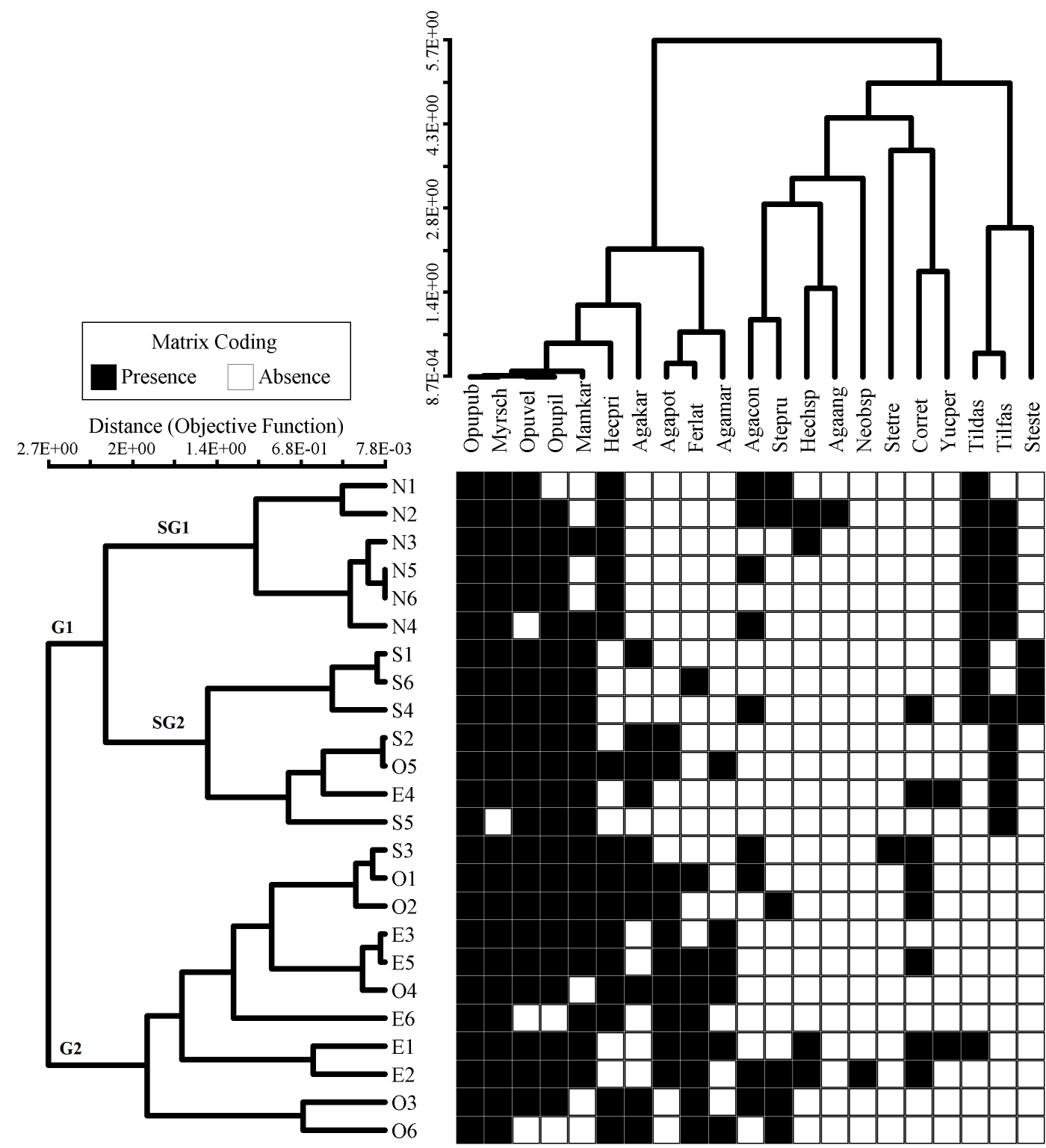

Figura 4. Agrupamiento de 24 unidades de muestreo y 21 especies de Yagul, Tlacolula, Oaxaca, con base en el índice de semejanza de Jaccard. Agaang= Agave angustifolia, Agacon $=$ A. convallis, Agakar $=$ A. karwinskii, Agamar $=$ A. marmorata, Agapot $=$ A. potatorum, Yucper $=$ Yucca periculosa , Hecpri $=$ Hechtia pringlei, Hechsp $=$ Hechtia sp., Tildas $=$ Tillandsia dasyliriifolia, Tilfas $=$ T. fasciculata, NCorret $=$ Coryphantha retusa, Ferlat $=$ Ferocactus latispinus, Mamkar = Mammillaria karwinskiana, Myrsch $=$ Myrtillocactus schenckii, Neobsp $=$ Neobuxbaumia sp., Opupil $=$ Opuntia pilifera, Opupub $=$ O. pubescens, Opuvel $=$ O. velutina, Stepru $=$ Stenocereus pruinosus, Steste $=$ S. stellatus, Stetre $=$ S. treleasei. 


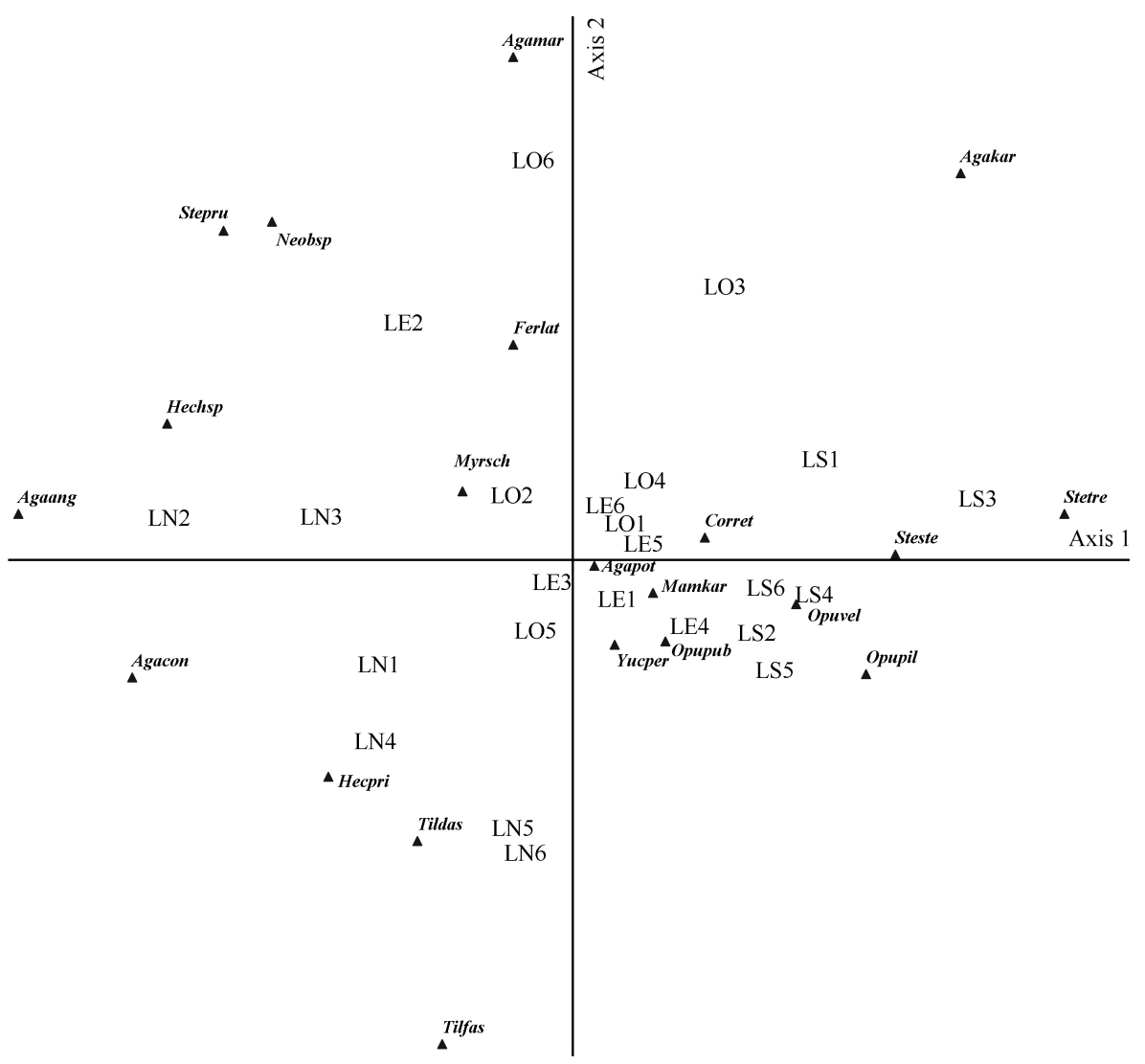

Figura 5. Análisis de Correspondencia de 24 unidades de muestreo y 21 especies de Yagul, Tlacolula, Oaxaca. Agaang= Agave angustifolia, Agacon $=$ A. convallis, Agakar $=$ A. karwinskii, Agamar $=$ A. marmorata, Agapot $=$ A. potatorum, Yucper $=$ Yucca periculosa, Hecpri $=$ Hechtia pringlei, Hechsp $=$ Hechtia sp., Tildas $=$ Tillandsia dasyliriifolia, Tilfas $=T$. fasciculata, Corret $=$ Coryphantha retusa , Ferlat $=$ Ferocactus latispinus, Mamkar = Mammillaria karwinskiana, Myrsch = Myrtillocactus schenckii, Neobsp = Neobuxbaumia $\mathrm{sp}$. , Opupil $=$ Opuntia pilifera, Opupub $=$ O. pubescens, Opuvel $=$ O. velutina, Stepru $=$ Stenocereus pruinosus, Steste $=$ S. stellatus, Stetre $=$ S. treleasei .

Tabla 3. Resultados del análisis de correspondencia canónica entre variables edáficas y datos de comunidades vegetales de 24 UM en laderas de Yagul, Tlacolula, Oaxaca.

\begin{tabular}{lccc}
\hline Ejes & $\mathbf{1}$ & $\mathbf{2}$ & $\mathbf{3}$ \\
\hline Valor propio & 0.561 & 0.434 & 0.358 \\
Varianza/Datos/Especie & & & \\
Varianza explicada (\%) & 12.6 & 9.7 & 8 \\
Varianza explicada acumulada (\%) & 12.6 & 22.3 & 30.3 \\
Correlación Especies-Ambiente & 0.948 & 0.905 & 0.893 \\
Correlación de variables con los ejes de ordenación & & & \\
conductividad eléctrica & 0.591 & -0.196 & -0.595 \\
pH & 0.387 & 0.546 & -0.365 \\
materia orgánica & 0.655 & 0.404 & 0.127 \\
nitrógeno total & 0.171 & 0.123 & -0.353 \\
fósforo & 0.745 & 0.116 & 0.007 \\
potasio & 0.403 & 0.242 & 0.541 \\
carbonato de calcio & 0.502 & 0.321 & -0.084 \\
\hline
\end{tabular}


Las correlaciones entre las variables y los ejes de ordenación (Figura 6), indican que, el eje uno diferencia las laderas con orientación sur, debido a que son las que tienen un mayor contenido de fósforo y materia orgánica, con valores promedio de $22.12 \mathrm{ppm}$ y $3.21 \%$ respectivamente. En las otras laderas, el contenido de ambos fue: Ladera Norte $=7.59$ ppm, $2.80 \%$, Ladera Este $=6.97 \mathrm{ppm}, 1.12 \%$ y Ladera Oeste $=16.16 \mathrm{ppm}, 0.31 \%$ para el fósforo y la materia orgánica, respectivamente. Las condiciones edáficas mencionadas para la ladera sur parecen ser las adecuadas para S. stellatus y Stenocereus treleasei (Vaupel) Backeb. El eje dos se correlacionó principalmente con el $\mathrm{pH}$ y los mayores valores se alcanzaron en las laderas $\operatorname{sur}(\chi=6.72)$ y este $(\chi=6.50)$, donde coexisten diversas especies de Opuntia.

\section{Discusión}

Los géneros con mayor número de especies fueron Agave, Stenocereus y Opuntia con 5, 3 y 3 respectivamente, esto coincide con reportes de Villaseñor (2004) sobre dichos géneros, en una relación que se manejó con mayor número de especies en México. Esto se deduce a que, en Yagul, se establece una gran diversidad de plantas vasculares.

El número de especies de suculentas encontradas en el sitio de Yagul, especialmente de Cactaceae, es similar a los de otros trabajos efectuados en otras zonas de Oaxaca y Puebla. En Yagul se registraron 12 especies de Cactaceae, cifra cercana a los informes de Guízar-Nolazco et al. (2005) en Tehuacán, Puebla, que fue menos de 17 especies, de López (2008) en Huajuapán de León, Oaxaca y Solano (2009) en Asunción, Cuyotepeji, Oaxaca. En contraste al número de especies registradas en Yagul, Reyes (1993) encontró en San Juan Mixtepec, Oaxaca, 14 especies de cactáceas y Tovar-Romero (2005) menciona 19 especies de Cactaceae para la región de Nizanda, Oaxaca. Además, la mayoría de estas especies se han documentado de regiones cercanas, en especial la Mixteca Alta. Por ejemplo, ambas zonas comparten ocho especies de Cactaceae: C. retusa, $F$. latispinus, $M$. karwinskiana perteneciente al género Mammillaria que posee la mayor riqueza de especies según

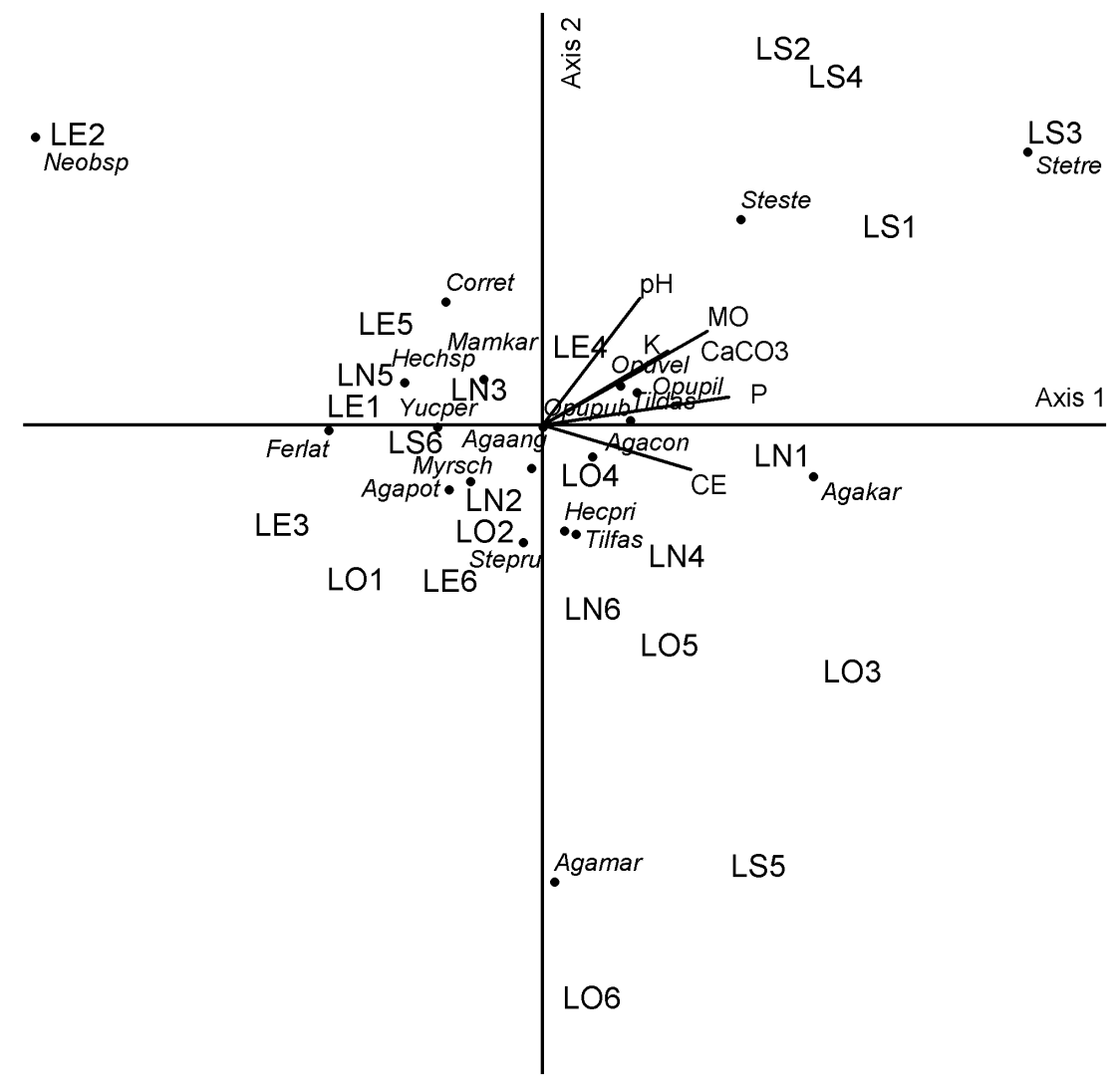

Figura 6. Diagrama de ordenación de especies suculentas de Yagul, Tlacolula, Oaxaca, obtenido con el método de Análisis Canónico de Correspondencias: Agaang= Agave angustifolia, Agacon $=A$. convallis, Agakar $=$ A. karwinskii, Agamar $=$ A. marmorata, Agapot $=A$. potatorum, Yucper $=$ Yucca periculosa , Hecpri $=$ Hechtia pringlei, Hechsp $=$ Hechtia sp., Tildas = Tillandsia dasyliriifolia, Tilfas $=T$. fasciculata, Corret $=$ Coryphantha retusa, Ferlat $=$ Ferocactus latispinus, Mamkar = Mammillaria karwinskiana, Myrsch = Myrtillocactus schenckii, Neobsp = Neobuxbaumia sp., Opupil = Opuntia pilifera, Opupub $=$ O. pubescens, Opuvel $=$ O. velutina, Stepru $=$ Stenocereus pruinosus, Steste $=$ S. stellatus, Stetre $=$ S. treleasei 
la NOM-059-SEMARNAT-2010 (SEMARNAT 2010), $M$. schenckii, $O$. pilifera, $O$. pubescens, $O$. velutina y S. stellatus una de las especies del género Stenocereus el cual radica su importancia puesto que el Servicio Nacional de Inspección y Certificación de Semillas (SNICS 2018) reportó de 17 a 24 especies y para nuestro país al menos 20 son endémicas; en particular para Yagul son tres especies del mencionado género lo que resalta su estatus ecológico. Esto muestra que, aunque el estudio se hizo en una superficie relativamente limitada, tiene una buena representación de la flora regional de suculentas. Cabe mencionar que los géneros Mammillaria y Coryphantha se encuentran al menos el 30 por ciento de sus especies en la NOM-059-SEMARNAT-2010. Con base en la norma anterior, el género con mayor cantidad de especies listadas en cierta categoría de riesgo es Mammillaria. Esto nos hace pensar en la importancia de estas especies en la zona de estudio. Un dato relevante es que Yagul se encuentra en un punto intermedio entre Cuicatlán y Tehuantepec, los cuales según Arias-Montes (1993) son los dos sitios de mayor riqueza de cactáceas en Oaxaca, por lo tanto, se resalta la importancia de Yagul como hábitat de las mencionadas especies vegetales.

La fisonomía de las formas biológicas columnares y arborescentes de M. schenckii, Stenocereus pruinosus (Otto ex Pfeiff.) Buxb., S. stellatus, $S$. treleasei, Neobuxbaumia sp. permiten diferenciar con claridad las comunidades vegetales de otras en Yagul, a pesar de que sus coberturas son reducidas. La única excepción es M. schenckii, conocido como "garambullo", que tiene amplias poblaciones a lo largo de las laderas. También se apreciaron especies favorecidas quizás por la perturbación tales como $O$. pubescens, $M$. karwinskiana y $O$. pilifera. Ferocactus latispinus, se halló principalmente asociada a rocas en las diferentes partes de la ladera, esta es una asociación muy semejante a la mencionada por HuertaMartínez \& Escobar-Santos (1998) y Escobar-Santos \& Huerta-Martínez (1999) para Ferocactus histrix; estos autores también mencionan que esta relación es debida al nodrizaje que le otorgan las rocas a $F$. histrix, lo cual, es probable que también ocurra en el caso de Ferocactus latispinus. Los cladodios de $O$. pubescens poseen numerosas espinas por lo que es una especie no consumida por fauna o ganado y es probable que las poblaciones de este taxón no se encuentran amenazadas. Otra población en la que se debe hacer énfasis es la de $C$. retusa (biznaga partida mocha); es un taxón restringido en ciertos sitios. La especie se observó en las partes bajas de los cerros, en la zona de lomeríos y terrenos planos y en suelos profundos derivados de rocas sedimentarias y pedregosas. Para el caso particular de A. karwinskii, conocido como "cuishe" se distribuyen grandes poblaciones silvestres en las laderas de Yagul.
Se observó un claro efecto de la orientación de las laderas. En parte, se confirmaron resultados previos, que mostraron que, en el lado norte, por ser ligeramente más húmeda (dato no probado), tenía más especies, que requieren algo de humedad ambiental, por ejemplo, las especies de la familia Bromeliaceae (Tillandsia achyrostachys, T. ionantha, T. dasyliriifolia y T. fasciculata) es necesario destacar la relevancia del género Tillandsia ya que es uno de los más representativos en México (Villaseñor 2004) y por ende para Yagul. Mientras que en la exposición sur dominan las especies rosetófilas con adaptaciones a sequía y las cactáceas globosas. Los lados oeste y este tenían cierta relación intermedia, como era de esperarse. La mayoría de las investigaciones previas comparan las exposiciones norte y sur de las laderas. En este trabajo se agregan las del este y oeste para evaluar qué tanto influyen dichas orientaciones en la estructura florística de las suculentas de Yagul.

En la región de Yagul, la frecuencia de formas rosetófilas aumenta en las laderas del sur, lo cual coincide con el estudio de Valverde-Padilla (2002). En las laderas orientadas hacia el este y el oeste de Yagul, se registró el mayor número de especies de suculentas, lo cual coincide con el estudio de Torres et al. (2012), no obstante, los autores reportan especies leñosas con mayor frecuencia en la ladera oeste que en la del este por lo que la exposición es uno de los factores que modifica la estructura florística y cobertura de la vegetación.

La variación en la composición y cobertura de las suculentas registradas en las diferentes posiciones geomorfológicas, tuvo una estrecha relación con las especies, debido a que en las laderas orientadas hacia el este, influye el mayor tiempo de exposición al sol (Pirela 2006) como factor que probablemente favorece la riqueza de suculentas, para el caso de Yagul, de especies tales como M. schenckii, F. latispinus, C. retusa, O. pubescens y Y. periculosa.

En Yagul, las especies se ubicaban a mayores altitudes en laderas de exposición este. Por ejemplo el A. potatorum, fue el único maguey que se encontró en lo más alto de las laderas de esta orientación. Era notable que sus dimensiones aumentaban en comparación a las que se encontraban en la parte media de las laderas, lo mismo ocurrió con F. latispinus. En contraste con laderas orientadas hacia el oeste, se registraron especies como A. karwinskii y S. pruinosus que tenían preferencia por las partes bajas.

El género Opuntia estuvo presente en todas las laderas estudiadas en Yagul, hecho que nos confirma que es uno de los géneros que cuenta con más especies (120) documentado por Villaseñor (2004); caso contrario de otras especies como $C$. retusa, F. latispinus y $Y$. periculosa, que fueron registrados solo en algunas laderas, es decir, su distribución tiende a ser más restringida. Esto es semejante 
a un estudio hecho en Zacatecas, en una nopalera en sitios contrastantes en exposición norte y sur, que presentaron especies dominantes presentes en el lado norte pero ausentes en los expuestos al sur (Del Castillo 2000).

En esta investigación se demostró que la exposición de la ladera, se relacionó con la densidad de plantas suculentas. Por lo que, la mayor presencia estuvo orientada al sur y al oeste. Posiblemente la disposición se deba a la tolerancia de las especies al calor y baja disponibilidad de agua. En otros estudios, señalan que las laderas orientadas al norte tienen escasas especies de cactos y suculentas (Niering et al. 1963, Woodhouse et al. 1980).

El análisis canónico de correspondencia destacó la importancia de materia orgánica y fósforo en la diferencia de las UM de Yagul y la flora asociada, principalmente de las laderas sur. Esto apoya resultados previos de GarcíaSánchez et al. (2012) y Sánchez-Soto et al. (2016) quienes también identificaron a estos elementos como importantes en la distribución de las especies (no exclusivamente suculentas) en sus sitios de estudio. Por otro lado, hubo una alta concentración de fósforo en el suelo de Yagul debido a que el mencionado nutrimento es producto de la descomposición de la materia orgánica (Montaño-Arias et al. 2006).

Es importante mencionar la relación entre el $\mathrm{pH}$ del suelo y el contenido de materia orgánica (MO), pues al descomponerse esta materia acidifica ligeramente el suelo, lo cual lleva a mejores condiciones de crecimiento en suelos alcalinos (Frías-Hernández et al. 1999); esto es demostrado por los coeficientes de correlación mayores con los ejes de ordenación de estos elementos en Yagul. De manera similar, Flores et al. (2007) reportaron éstas mismas variables para un estudio realizado en el altiplano central (Aguascalientes y el norte de Jalisco) los cuales tienen una influencia positiva en las especies vegetales. Los mayores porcentajes de MO podrían atribuirse a la acumulación de biomasa entre las rocas y en las grietas a nivel de la superficie del suelo (Mazzola et al. 2008). Desde el punto de vista edáfico, en Yagul se observaron suelos someros y pedregosos en la mayor parte del área, con diferentes niveles de alcalinidad y salinidad producto de la influencia de los diferentes substratos geológicos presentes en la zona de estudio. Estas características del suelo de Yagul son similares a las de Zapotitlán de las Salinas, Puebla (López-Galindo et al. 2003). También, la alta concentración de materia orgánica, puede ser explicada como el resultado de la acumulación de la hojarasca, como lo describen Rostagno et al. (1991), esto se observó continuamente durante el muestreo de suelo ya que en estos se encontraba hojarasca en descomposición, principalmente de especies de la familia Fabaceae.

El análisis de ordenación de la comunidad indica que las variables asociadas al substrato donde crecen las suculentas, junto con la exposición de las laderas de Yagul, son importantes en la distribución de las especies estudiadas. Esto resalta la necesidad de estudiar más detalladamente estas laderas, que ocupan superficies considerables en dicha área, con la finalidad de evaluar su contribución a la diversidad florística.

Las variables edáficas como cobertura de rocas y profundidad del suelo son factores marcados en la zona de Yagul, debido a que ejercen influencia en la distribución y abundancia de las especies. También se ha informado en otras localidades secas o demasiado secas del país (HuertaMartínez \& García-Moya 2004, Huerta-Martínez et al. 2004).

Con base en esta investigación, se propone conservar a C. retusa ya que es una de las especies que pertenece al género Coryphantha y éste ocupa el cuarto lugar de los que tienen más especies en el hemisferio norte de acuerdo a la NOM-059-SEMARNAT-2010 (SEMARNAT 2010), además de que es endémica y se encuentra en la categoría de riesgo sujeta a protección especial. También otras especies como F. latispinus, S. pruinosus y S. treleasei debido a que son escasas. Para una futura repoblación de las especies, se propone considerar, las condiciones altitudinales, edáficas y de orientación.

\section{Agradecimientos}

Al Consejo Nacional de Ciencia y Tecnología (CONACyT) por la beca otorgada a JCLM para estudios de Maestría en Ciencias (781551/616456). A las autoridades del Área Natural Protegida "Monumento Natural Yagul", por permitir llevar a cabo el trabajo de campo. MLC agradece a Angélica Romero-Manzanares la lectura crítica y observaciones al manuscrito

\section{Literatura citada}

APG [The Angiosperm Phylogeny Group], Chase MW, Christenhusz MJM, Fay MF, Byng JW, Judd WS, Soltis DE, Mabberley DJ, Sennikov AN, Stevens PF. 2016. An update of the Angiosperm Phylogeny Group classification for the orders and families of flowering plants. Botanical Journal of the Linnean Society 181: 1-20. DOI: https://doi.org/10.1111/boj.12385

Arias-Montes S. 1993. Cactáceas: conservación y diversidad en México. Diversidad biológica en México. Revista de la Sociedad Mexicana de Historia Natural 44: 109-115.

Bremmer JM, Mulvaney CS. 1982. Total nitrogen. Part 1 Methods of soil analysis. Part 2 Chemical and microbiological properties. Madison, Wisconsin, USA: The American Society of Agronomy (ASA).

CONABIO [Comisión Nacional para el Conocimiento y Uso de la Biodiversidad]. 2008. Capital natural de 
México, vol. I: Conocimiento actual de la biodiversidad. México, DF: Comisión Nacional para el Conocimiento y Uso de la Biodiversidad. ISBN 978-607-7607-03-8.

Del Castillo RF. 1987. Efectos del Disturbio y la Orientación de Ladera en Ferocactus histrix. Cactáceas y Suculentas Mexicanas 32: 8-16.

Del Castillo RF. 2000. Composición y estructura de una nopalera bajo situaciones contrastantes de exposición de ladera y herbivoría. Boletín de la Sociedad Botánica de México 65: 5-22. DOI: https://doi.org/10.17129/botsci. $\underline{1591}$

Escobar-Santos V, Huerta-Martínez FM. 1999. Relaciones ecológicas de Ferocactus histrix (DC.) Lindsay en los llanos de Ojuelos, Jalisco-Zacatecas. Cactáceas y Suculentas Mexicanas 44: 40-48.

Flores E, Frías J, Jurado P, Olalde V, Figueroa JD, Valdivia A, García E. 2007. Efecto del gatuño sobre la fertilidad del suelo y la biomasa herbácea en pastizales del centro de México. Terra Latinoamericana 25: 311-319.

Frías-Hernández JT, Aguilar-Ledezma AL, Olalde-Portugal V, Balderas-López JA, Gutiérrez-Juárez G, Alvarado-Gil JJ, Castro JJ, Vargas H, Albores A, Dendooven L, Miranda L. 1999. Research note soil characteristics in semiarid highlands of Central Mexico as affected by mesquite trees (Prosopis laevigata). Arid Soil Research and Rehabilitation 13: 305-312. DOI: https://doi.org/ 10.1080/089030699263339

García E. 2004. Modificaciones al sistema de clasificación climática de Köppen. México, DF: Instituto de Geografía, Universidad Nacional Autónoma de México. ISBN: 970-32-1010-4

García-Sánchez R, Camargo-Ricalde SL, García-Moya E, Luna-Cavazos M, Romero-Manzanares A, Montaño NM. 2012. Prosopis laevigata and Mimosa biuncifera (Leguminosae), jointly influence plant diversity and soil fertility of a Mexican semiarid ecosystem. Revista de Biología Tropical 60: 87-103. DOI: https://doi.org/ $\underline{10.15517 / \mathrm{rbt} . v 60 \mathrm{i} 1.2365}$

Guízar-Nolazco E, Mota-Cruz C, Ortega-Paczka R. 2005. Vegetación y plantas útiles en la subregión Filo de Tierra Colorada, Reserva de la Biosfera Tehuacán-Cuicatlán, México. Revista de Geografía Agrícola 35: 67-84.

Hill MO, Gauch Jr. HG. 1980. Detrended correspondence analysis, an improved ordination technique. Vegetatio 42 : 47-58. DOI: https://doi.org/10.1007/978-94-009-919727

Huerta-Martínez FM, Escobar-Santos VE. 1998. Estatus ecológico actual de Ferocactus histrix (DC) Lindsay en los llanos de Ojuelos, Jalisco-Zacatecas. Cactáceas y Suculentas Mexicanas 43: 57-63.
Huerta-Martínez FM, García-Moya E. 2004. Diversidad de especies perennes y su relación con el ambiente en un área semiárida del centro de México: implicaciones para su conservación. Interciencia 29: 435-441.

Huerta-Martínez FM, Vázquez-García JA, García-Moya E, López-Mata L, Vaquera-Huerta H. 2004. Vegetation ordination at the southern Chihuahuan Desert (San Luis Potosí, Mexico). Plant Ecology 174: 79-87. DOI: https:// doi.org/10.1023/B:VEGE.0000046063.12313.74

INEGI [Instituto Nacional de Estadística y Geografía]. 2000. Carta de uso actual del suelo y vegetación. Serie III, Escala 1:250,000. México, DF: Instituto Nacional de Estadística y Geografía.

INEGI. 2005. Mapa de climas, escala 1:1 000 000. https:// www.inegi.org.mx/temas/climatologia/ (consultado Septiembre 26, 2019).

López MJ. 2008. Estudio etnobotánico en el municipio de Santo Domingo Yodohino, distrito de Huajuapan de León, Oaxaca. BSc. Thesis, Universidad Autónoma Chapingo, México.

López-Galindo F, Muñoz-Iniestra D, Hernández-Moreno M, Soler-Aburto A, Castillo-López MC, HernándezArzate I. 2003. Análisis integral de la toposecuencia y su influencia en la distribución de la vegetación y la degradación del suelo en la Subcuenca de Zapotitlán Salinas, Puebla. Boletín de la Sociedad Geológica Mexicana 56: 19-41. DOI: http://dx.doi.org/10.18268/ BSGM2003v56n1a3

López-Gómez V, Zedillo-Avelleyra P, Anaya-Hong S, González-Lozada E, Cano-Santana Z. 2012. Efecto de la orientación de la ladera sobre la estructura poblacional y ecomorfología de Neobuxbaumia tetetzo (Cactaceae). Botanical Sciences 90: 453-457. DOI: https://doi.org/ 10.17129/botsci.473

Lot A, Chiang F. 1986. Manual de Herbario. Administración y Manejo de Colecciones, Técnicas de Recolección y Preparación de Ejemplares Botánicos. México, DF: Consejo Nacional de la Flora de México. ISBN: 9686144005

Mata-González R, Pieper RD, Cárdenas MM. 2002. Vegetation patterns as affected by aspect and elevation in small desert mountains. The Southwestern Naturalist 47: 440-448. DOI: https://doi.org/10.2307/3672501

Mazzola MB, Kin AG, Morici EF, Babinec FJ, Tamborini G. 2008. Efecto del gradiente altitudinal sobre la vegetación de las sierras de Lihue Calel (La Pampa, Argentina). Boletín de la Sociedad Argentina de Botánica 43: 103-119.

McCune B, Grace JB. 2002. Analysis of Ecological Communities. Gleneden Beach, Oregon: MjM Software Design. 
McCune B, Mefford MJ. 2011. PC-ORD v. 6. Multivariate Analysis of Ecological Data. Gleneden Beach, Oregon: MjM Software Design . ISBN-10: 0972129006; ISBN-13: 978-0972129008

Mielke PW. 1991. The application of multivariate permutation methods based on distance functions in the earth sciences. Earth-Science Reviews 31: 55-71. DOI: https://doi.org/10.1016/0012-8252(91)90042-E

Mielke PW, Berry KJ. 1976. Multi-response permutation procedures for a priori classifications. Communications in Statistics - Theory and Methods 5:1409-1424. DOI: https://doi.org/10.1080/03610927608827451

Miranda F, Hernández X. 2013. Los tipos de vegetación de México y su clasificación. Edición Conmemorativa 1963-2013. Mexico, DF: Comisión Nacional para el Conocimiento y Uso de la Biodiversidad (CONABIO) / Fondo de Cultura Económica (FCE): ISBN: 9786071618634

Montaño-Arias NM, García-Sánchez R, Ochoa-de la Rosa G, Monroy-Ata A. 2006. Relación entre la vegetación arbustiva, el mezquite y el suelo de un ecosistema semiárido en México. Terra Latinoamericana 24: 193-205.

Mostacedo B, Frederiksen T. 2000. Manual de métodos básicos de muestreo y análisis en ecología vegetal. Santa Cruz, Bolivia: Editora El País.

Niering WA, Whittaker RH, Lowe CH. 1963. The saguaro: a population in relation to environment. Science 142: 15-23. DOI: https://doi.org/10.1126/science.142.3588.15

Peck JE. 2010. Multivariate analysis for community ecologists: Step by step using PC-ORD. Gleneden Beach, Oregon, USA: MjM Software Design. ISBN 978-0-9721290-2-2

Pirela M. 2006. Análisis Funcional de la Comunidad de Plantas en tres unidades geomorfológicas del Páramo de Mucubají. BSc. Thesis, Universidad de los Andes, Venezuela.

Reyes J. 1993. Estudio florístico y fitogeográfico en el municipio de San Juan Mixtepec, distrito de Juxtlahuaca, Oaxaca. BSc. Thesis, Universidad Nacional Autónoma de México.

Reyes-Olivas A, García Moya E, Terrazas Salgado T, López Mata L., Vaquera Huerta H, Valiente Banuet A. 2002.Patrones espaciales de cactáceas en el desierto costero de Topolobampo. PhD. Thesis, Colegio de Postgraduados, Campus Montecillo, Instituto de Recursos Naturales.

Rostagno CM, Del Valle HF, Videla L. 1991. The influence of shrubs on some chemical and physical properties of an aridic soil in north-eastern Patagonia, Argentina. Journal of Arid Enviroments 20: 179-188. DOI: https://doi.org/ 10.1016/S0140-1963(18)30707-9

Rzedowski J. 1978. Vegetación de México. México, DF: Editorial Limusa. ISBN: 968-18-0002-8

Sánchez-Soto BH, García-Moya E, Reyes-Olivas Á, Romero-Manzanares A, Luna-Cavazos M. 2016. Factores topográficos y edáficos que influyen en la estructura de especies perennes de islas de la costa de Sinaloa, México. Botanical Sciences 94: 63-73. DOI: https://doi.org/10.17129/botsci.219

SEMARNAT. [Secretaría del Medio Ambiente y Recursos Naturales]. 2002. Norma Oficial Mexicana NOM-021RECNAT-2000, Especificaciones de Fertilidad, Salinidad y Clasificación de Suelos, Estudio, Muestreo y Análisis. Diario Oficial de la Federación. 2da Sección, 31 de diciembre de 2002.

SEMARNAT. 2010. Norma Oficial Mexicana NOM-059SEMARNAT-2010, Protección ambiental - Especies nativas de México de flora y fauna silvestres - Categorías de riesgo y especificaciones para su inclusión, exclusión o cambio - Lista de especies en riesgo. Diario Oficial de la Federación. 2da Sección, 30 de diciembre de 2010.

SEMARNAT. 2013. Programa de manejo del Monumento Natural Yagul. México, DF: Secretaría de Medio Ambiente y Recursos Naturales y Pesca. ISBN 978-607-8246-54-0

SNICS [Servicio Nacional de Inspección y Certificación de Semillas]. 2018. Diagnóstico de la pitaya (Stenocereus spp.). https://www.gob.mx/snics/acciones-y-programas/ diagnostico-de-la-pitaya-stenocereus-spp-en-mexico (Consultado: octubre 23, 2019).

Solano HL. 2009. Importancia ecológica y cultural de los recursos vegetales de Asunción Cuyotepeji, Oaxaca, México. MsC. Thesis, Centro Interdisciplinario de Investigación para el Desarrollo Integral Regional Oaxaca, México.

Ter Braak CJ. 1986. Canonical correspondence analysis: a new eigenvector technique for multivariate direct gradient analysis. Ecology 67: 1167-1179. DOI: https:// doi.org/10.2307/1938672

Torres E, Schwarzkopf T, Fariñas M, Aranguren A. 2012. ¿Es la orientación de la pendiente un factor modificador de la estructura florística en la alta montaña tropical andina? Ecotrópicos 25: 61-74.

Tovar-Romero HA. 2005. Morfología y distribución de las cactáceas en el paisaje complejo de Nizanda (Oaxaca). BsC. Thesis, Universidad Nacional Autónoma de México.

Valverde-Padilla PL. 2002. Cambios en la abundancia, dominancia y diversidad de formas de vida vegetales 
entre laderas norte y sur en el Valle semiárido de Zapotitlán, Puebla. ContactoS 45: 45-50.

Villaseñor JL. 2004. Los géneros de plantas vasculares de la flora de México. Boletín de la Sociedad Botánica de México 75: 105-135. DOI: https://doi.org/10.17129/ botsci. 1694

Walkley A, Black IA. 1934. An examination of the Degtjareff method for determining soil organic matter,

Editor de sección: Enrique Jurado

Contribución de los autores: JCLM diseñó el estudio, llevó a cabo el trabajo de campo, analizó los datos y escribió el manuscrito. MLC, diseñó el estudio, asesoró el trabajo, analizó los datos y escribió el manuscrito, contribuyó a la discusión y revisión del manuscrito. HV, diseñó el estudio, asesoró el trabajo, contribuyó a la redacción, discusión y revisión del manuscrito. MFC, asesoró el trabajo en general, contribuyó a la discusión y revisión del manuscrito. and a proposed modification of the chromic acid titration method. Soil Science 37: 29-38.

Woodhouse RM, Williams JG, Nobel PS. 1980. Leaf orientation, radiation interception and nocturnal acidity increases by the CAM plant Agave deserti (Agavaceae). American Journal of Botany 67: 1179-1185. DOI: https:// doi.org/10.1002/j.1537-2197.1980.tb07751.x 